\title{
In vitro toxicity evaluation of graphene oxide on human RPMI 8226 cells
}

\author{
Yuzhen Wang ${ }^{\mathrm{a}}$, Shaoling $\mathrm{Wu}^{\mathrm{a}, *}$, Xindong Zhao ${ }^{\mathrm{b}}, \mathrm{Zhan} \mathrm{Su}^{\mathrm{a}}, \mathrm{Li} \mathrm{Du}^{\mathrm{a}}$ and Aihua Sui ${ }^{\mathrm{a}}$ \\ ${ }^{a}$ Department of Hematology, The Affiliated Hospital of Medical College Qingdao University, 16 \\ Jiangsu Road, Qingdao, Shandong 266003, China \\ ${ }^{b}$ Department of Hematology, Medical College of Qingdao University, 38 Dengzhou Road, Qingdao, \\ Shandong 266021, China
}

\begin{abstract}
This study had investigated the possible toxicity of graphene oxide and its mechanisms on multiple myeloma cells (RPMI 8226 cells) using flow cytometry and a multifunctional microplate reader. RPMI 8226 cells were cultured with various concentrations of graphene oxide, then cell viability, malondialdehyde, glutathione and apoptosis were measured. We found that graphene oxide dose-dependently reduced the viability of human multiple myeloma RPMI 8226 cells. We also found that the intracellular levels of malondialdehyde increased, whereas the levels of glutathione decreased dose-dependently. There was no obvious change in the cell apoptosis rate compared with the control group. In summary, graphene oxide is dose-dependently cytotoxic to cultured RPMI 8226 cells, and its toxicity is closely associated with increased oxidative stress.
\end{abstract}

Keywords: Graphene oxide, cytotoxicity, oxidative stress, RPMI 8226 cells

\section{Introduction}

Graphene oxide (GO) is a nanomaterial composed of carbon atoms in a one-atom-thick monolayer, with a two-dimensional honeycomb structure. Because of its unique physical and chemical properties, GO has attracted a great deal of interest in medicine [1] and has been extensively investigated for biomedical applications including cancer therapies [2], molecular probes [3], disease diagnosis [4], bioimaging [5], and biosensors [6]. Many studies have shown that GO is a nanocarrier that can be loaded with anticancer drugs $[7,8]$. This is highly relevant for the advancement of nanomedicine, a very hot research area for future therapy. However, the clinical application of nanomaterials [9], and especially carbon nanomaterials [10] still faces many challenges. One for all, the need for more extensive cytotoxic studies provides sufficient data for a comprehensive view and advancement of the field [11].

With the increaing development of GO for biomedical applications, it is inevitable that humans will contact GO in the living environment. Consequently, it is critical to evaluate that its potential risk to human health be systematically evaluated. Several pioneers have studied the cytotoxicity of GO in

\footnotetext{
* Corresponding author: Shaoling Wu, Department of Hematology, The Affiliated Hospital of Medical College Qingdao University, 16 Jiangsu Road, Qingdao, Shandong 266003, China. Tel.: +86 532-82911393; E-mail: qdwushaoling@163.com.
} 
healthy cell lines, including human mesenchymal stem cells [12], fibroblasts $[13,14]$ and erythrocytes [15]. Their results suggested that GO was cytotoxic to healthy cells; however, caution must be taken before making generalizations. Cytotoxicity strongly depends on the specific graphene or GO material used, e.g. its purity, physical properties, and kind and level of chemical functionalization [16-18].Several studies have also addressed the toxic effects of GO in tumor cells. These results may be attributable to the increased generation of reactive oxygen species (ROS) and the high cellular uptake of GO [19]. However, some investigators [20] reported that GO did not enter A549 cells and exerted no significant cytotoxic effects, but induced oxidative stress in the cells at high concentrations. Lammel et al. demonstrated that GO was cytotoxic to HepG2 cells and that plasma membrane damage and oxidative stress were significant cytotoxic mechanisms in these cells [21]. Zhang et al. reported the cytotoxicity of graphene in PC12 cells [22]. Their study clearly demonstrated that ROS played important roles in this cytotoxicity. Thus, many researchers had reported the cytotoxicity of GO in different tumor cells and showed that oxidative stress played a key role in it.

The incidence of hematological malignancy has increased continuously in the last decade. A GO-based nano-drug could be used to treat hematological malignancies. However, few data concerning the application of nanomaterials to malignant hematological cells have been reported, no study has systematically investigated the cytotoxic effects and mechanisms of GO on multiple myeloma cells. The present study investigated the cytotoxicity of GO by examining cell viability using human multiple myeloma RPMI 8226 cells. The possible mechanisms were evaluated by malondialdehyde (MDA), glutathione (GSH) and apoptosis. This study provides detailed information about the cytotoxic effects of GO on multiple myeloma cells and offers a sound basis for the clarification of its toxicity mechanisms.

\section{Materials and methods}

\subsection{Materials}

Graphene oxide (GO-L-L-NA) was obtained from Nanoon Nanomaterials Technology Co., Ltd (Beijing, China). The lateral size of graphene oxide was smaller than $100 \mathrm{~nm}$. Graphene oxide purity was greater than 99.9\%. Fetal bovine serum (FBS) was acquired from Gibco Life technologies (Gaithersburg, USA). RPMI 1640 medium, penicillin and streptomycin were obtained from Beijing Zhongshan Golden Bridge Biotechnology CO., Ltd (Beijing, China). Cell Counting Kit-8 (CCK-8) assay kits were obtained from DojinDo laboratories (Kyushu, Japan). Malondialdehyde (MDA) assay kit, Glutathione (GSH) assay kits were purchased from Nanjing Jiancheng Bioengineering Institute (Jiangsu, China), Alexa Fluor ${ }^{\circledR} 488$ annexin V/Dead Cell Apoptosis Kit was purchased from Life Technologies (Carlsbad, USA).

\subsection{Cell culture}

RPMI8226 cells were obtained from American Type Culture Collection (CCL-155 ${ }^{\mathrm{TM}}$, Manassas, USA). Cells were maintained in cell culture flasks in RPMI-1640 medium supplemented with $10 \%$ (v/v) FBS, $100 \mathrm{U} / \mathrm{mL}$ penicillin and $100 \mu \mathrm{g} / \mathrm{mL}$ streptomycin at $37^{\circ} \mathrm{C}$ in a humidified atmosphere of $5 \% \mathrm{CO}_{2} / 95 \%$ air in a Thermo Scientific $3110 \mathrm{CO}_{2}$ incubator (Plymouth, USA). In all tests, the RPMI8226 cells in logarithmic growth phase were used to experiment. Cell morphology was observed using an optical microscope (Tokyo, Japan) after $24 \mathrm{~h} \mathrm{GO}$ exposure. 


\subsection{Cell viability assay}

Cell viability was evaluated by CCK- 8 method. RPMI 8226 cells were seeded in 96 well microplate at a density of $1.5 \times 10^{5}$ cells $/ \mathrm{mL}$ in $100 \mu \mathrm{L}$ each well, then cells were cultured in medium with various concentrations $(0,10,25,50,100,200 \mathrm{mg} / \mathrm{L})$ of GO for $24 \mathrm{~h}$. The cell-free with GO blank groups were performed to see if the GO reacted directly with the CCK-8 reagent. $10 \mu \mathrm{L}$ CCK-8 reagent was added to each well and incubated $2 \mathrm{~h}$ at $37^{\circ} \mathrm{C}$ under $5 \% \mathrm{CO}_{2}$, the optical density was measured at 450 $\mathrm{nm}$ using microplate reader (Tecan, Mannedorf, Switzerland). Cell viability was expressed as a percent of (OD test - OD blank)/(OD control - OD blank), where OD test was the optical density of the cells exposed to GO, OD control was the optical density of the cells without GO and OD blank was the optical density of cell medium with GO.

\subsection{Measurement of MDA}

RPMI 8226 cells were seeded into six-well microplates at $2.0 \times 10^{6}$ cells per well. After incubation with the indicated concentrations of GO $(0,10,25,50,100,200 \mathrm{mg} / \mathrm{L})$ for $24 \mathrm{~h}$, the cells were harvested and washed twice with ice-cold phosphate-buffered saline. The cells were collected and disrupted by ultrasonication for $5 \times 6 \mathrm{~s}$ with a $5 \mathrm{~s}$ pause in between using a sonicator probe (VCX-130 $\mathrm{W}$, Newtown, USA) on ice. The cell extract $(100 \mu \mathrm{L})$ was used to detect MDA according to the procedure recommended by the manufacturer of the MDA assay kit. The concentration of MDA was measured on a microplate reader (Tecan, Mannedorf, Switzerland) at a wavelength of $532 \mathrm{~nm}$. Coomassie Brilliant Blue method was used to quantify the protein in the cells.

\subsection{Measurement of glutathione}

The cell samples were treated with a method similar to that used to measure MDA. The concentration of GSH was expressed as nmol per milligram of protein. The cell suspension $(100 \mu \mathrm{L})$ was mixed with $100 \mu \mathrm{L}$ of precipitant and centrifuged at $4000 \times \mathrm{g}$ for $10 \mathrm{~min}$. After centrifugation, the supernatant $(100 \mu \mathrm{L})$ was used for the GSH assay, performed according to the manufacturer's protocol. The reaction was monitored at $405 \mathrm{~nm}$ in a microplate reader. Coomassie Brilliant Blue method was used to quantify the protein in the cells.

\subsection{Cell apoptosis analysis}

The RPMI 8226 cells were prepared in the concentration of $6.0 \times 10^{5} / \mathrm{mL}$ and seeded $2 \mathrm{~mL}$ in 6 well plate and treated with various concentrations of $\mathrm{GO}(0,10,50,100 \mathrm{mg} / \mathrm{L})$ cultured for $24 \mathrm{~h}$. Determined the cell density and diluted in annexin-binding buffer to $1 \times 10^{6}$ cells $/ \mathrm{mL}$. $100 \mu \mathrm{L}$ cells were moved to $1.5 \mathrm{~mL}$ EP tube and stained with $5 \mu \mathrm{L}$ annexin V-FITC and $1 \mu \mathrm{L}$ PI $(100 \mu \mathrm{g} / \mathrm{mL})$ without permeabilization. Then, RPMI 8226 cells were incubated at room temperature for 15 minutes. Finally, $400 \mu \mathrm{L}$ annexin-binding buffer was added into each sample and mixed well. Within one hour, the stained RPMI 8226 cells were analyzed by flow cytometry (CytomicTM FC500, Beckman coulter, USA). 


\subsection{Statistical analysis}

All data were presented as mean \pm Standard Deviation (SD) of a representative of three experiments carried out in triplicate. Statistical analysis was performed using the SPSS version 19 software. A $\mathrm{P}$-value $<0.05$ was taken as statistically significant.

\section{Results}

\subsection{Effects of GO on cell viability}

Effects of GO on cell morphology of RPMI 8226 cells were shown in Figure 1. We investigated the viability of RPMI 8226 cells that had been incubated with different concentrations of GO for $24 \mathrm{~h}$. As shown in Figure 2, cell viability decreased to $95 \%, 88 \%, 85 \%, 78 \%$, and $61 \%$ when the cells were exposed to GO at concentrations of $10,25,50,100$, and $200 \mathrm{mg} / \mathrm{L}$, respectively $(\mathrm{p}<0.05)$. When the cells were exposed to $10 \mathrm{mg} / \mathrm{L}$ GO, the reduction in cell viability was $5 \%$; when GO was increased to $200 \mathrm{mg} / \mathrm{L}$, the reduction in cell viability was approximately $40 \%$.
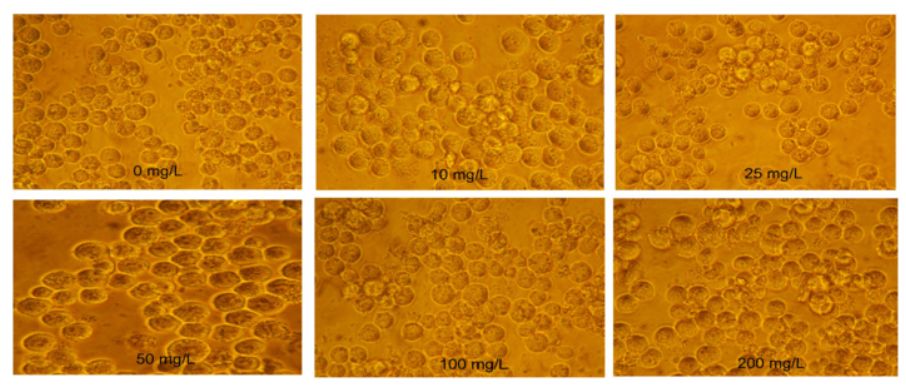

Fig. 1. Optical microscopy morphological characterization of RPMI 8226 cells were treated with various GO for $24 \mathrm{~h}$ at $37^{\circ} \mathrm{C}$. The images were visualized under an inverted microscope (400 X).

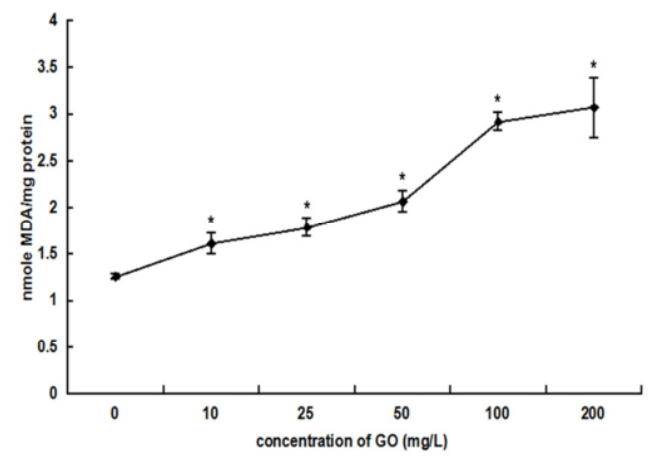

Fig. 3. Cellular MDA levels of RPMI 8226 cells exposure to $\mathrm{GO}$ at $37^{\circ} \mathrm{C}$ for $24 \mathrm{~h}$. Data were shown as mean $\pm \mathrm{SD}$. Statistically significant difference as compared to control * $(\mathrm{P}<0.05)$.

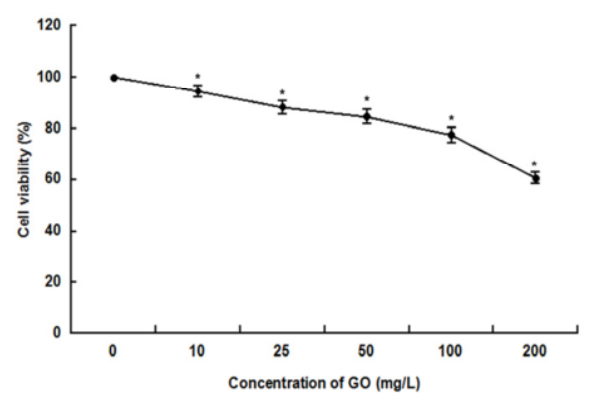

Fig. 2. Effects of GO on cell viability of RPMI 8226 cells exposure to $\mathrm{GO}$ at $37^{\circ} \mathrm{C}$ for $24 \mathrm{~h}$. Data were shown as mean $\pm \mathrm{SD}$. Compared with control, the difference was significant $*(\mathrm{P}<0.05)$.

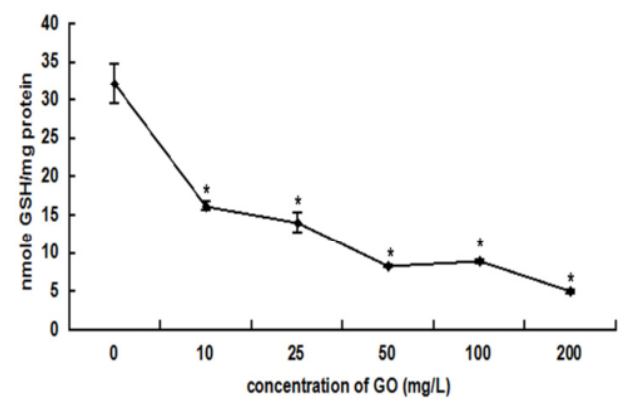

Fig. 4. Cellular GSH levels of RPMI 8226 cells at $37^{\circ} \mathrm{C}$ after $24 \mathrm{~h}$ exposure to GO. Data were shown as mean $\pm \mathrm{SD}$. There was significant difference as compared to control * $(\mathrm{P}<0.05)$. 


\subsection{Oxidative stress induced by $G O$}

To determine whether the cytotoxic effect of GO on RPMI 8226 cells was induced by oxidative stress, we assayed the cellular levels of MDA and GSH. The levels of MDA were shown in Figure 3. MDA increased from $1.6 \pm 0.1$ to $3.1 \pm 0.3 \mathrm{nmol} / \mathrm{mg}$ protein with exposure to $10-200 \mathrm{mg} / \mathrm{L}$ GO, whereas the MDA concentration in the control was $1.3 \pm 0.1 \mathrm{nmol} / \mathrm{mg}$ protein $(\mathrm{p}<0.05)$. These results suggested that GO significantly increased the intracellular production of MDA. The levels of GSH were shown in Figure 4. GSH decreased from $16.20 \pm 0.6$ to $5.0 \pm 0.3 \mathrm{nmol} / \mathrm{mg}$ protein after exposure to $10-200 \mathrm{mg} / \mathrm{L}$ GO, whereas the control level was $32.2 \pm 2.6 \mathrm{nmol} / \mathrm{mg}$ protein $(\mathrm{p}<0.05)$. These results suggested that GO significantly decreased intracellular GSH.

\subsection{Effects of $G O$ on apoptosis}

Apoptosis was detected with annexin V-FITC/PI staining and flow cytometry. The effects of GO on apoptosis were shown in Figure 5. After exposure to 0,10, 50, or $100 \mathrm{mg} / \mathrm{L} \mathrm{GO}$ for $24 \mathrm{~h}$, the apoptosis rates were $5.10 \%, 4.94 \%, 5.01 \%$, or $5.06 \%(\mathrm{P}=0.70, \mathrm{P}=0.11, \mathrm{P}=0.69)$, respectively. Thus, there was no significant difference in the apoptosis rate after treatment with the three different concentrations of GO and in the untreated cells.

\section{Discussion}

The potential influence of GO on biological safety and human health has attracted significant attention since GO was first successfully prepared in 2004. Some studies have examined the cytotoxicity of GO in different types of cell lines, but there is no report of the effects of GO on multiple myeloma cells. In this study, we used human multiple myeloma RPMI 8226 cells as a cell model for various assays of different toxicity endpoints to evaluate the potential cytotoxicity of GO and to clarify its underlying mechanisms.

The cell viability of RPMI 8226 cells was observed by CCK- 8 colorimetric assay after exposure to GO. The results suggested that GO was cytotoxic to RPMI 8226 cells and that effect was concentration dependent. The results were supported by those of other investigators, including Lammel et al. [21] who showed that GO had a dose-dependent cytotoxic effect on HepG2 cells, and Liao et al. [15], who found that the cytotoxicities of erythrocytes and skin fibroblasts increased with increasing concentration of GO. Further studies were needed to clarify the cytotoxic mechanisms of

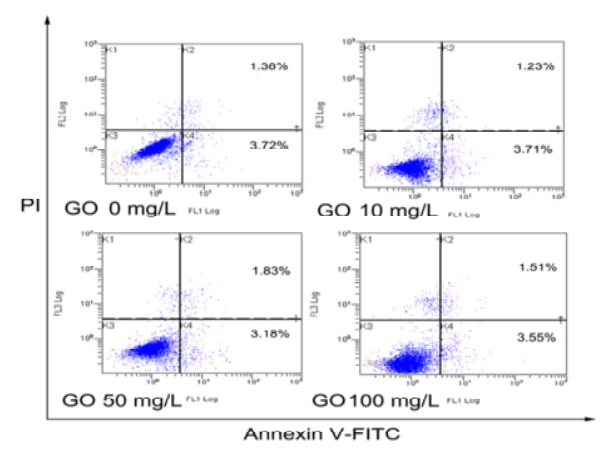

Fig. 5. The results of Annexin V-FITC/PI double stain about RPMI 8226 cells after 24 h GO exposure. 
GO. Oxidative stress might be an important mechanism responsible for the cytotoxicity of nanomaterials $[13,23]$. An imbalance between pro-oxidant (MDA) and antioxidant (GSH) compounds could lead to oxidative stress. In this study, oxidative stress was evaluated by the measurement of intracellular MDA and GSH levels. The levels of MDA indicated that lipid peroxidation significantly increased at GO concentrations of 10-200 $\mathrm{mg} / \mathrm{L}$. Interestingly, the levels of intracellular MDA in cells treated with $200 \mathrm{mg} / \mathrm{L} \mathrm{GO}$ were two-fold higher than those in cells treated with $10 \mathrm{mg} / \mathrm{L} \mathrm{GO}$. These results indicated that GO dose-dependently affected MDA levels. Surprisingly, the intracellular GSH in cells treated with $200 \mathrm{mg} / \mathrm{L}$ GO was 3.24-fold lower than those in cells treated with $10 \mathrm{mg} / \mathrm{L}$ GO. Thus, GO significantly and dose-dependently decreased intracellular GSH concentrations. The simultaneous GO-dose-dependent increased in MDA and decreased in GSH. Those indicated that the RPMI 8226 cells were experiencing oxidative stress. Briefly, the results demonstrated that GO stimulated the generation of oxidative stress and reduced cell viability. Several other studies have also reported that GO promoted cytotoxicity predominantly through generation of oxidative stress in malignant non-hematological cell lines [21,24]. Thus, the mechanisms of GO cytotoxicity could involve oxidative stress. Wang et al. reported that GO induced oxidative stress in human lung fibroblasts [13], and the results suggested that the oxidative stress was an effective mechanism involved in the cytotoxicity of GO. However, about multiple myeloma, the potential toxic effects of GO on human have not yet been studied. Therefore, more experimental studies are needed to characterize the mechanisms underlying the toxic effects of GO more precisely.

Cell apoptosis was also assessed with flow cytometry. There was no significant increase in the proportion of apoptotic cells after treatment with GO. The results showed that GO did not induce apoptosis, which was consistent with the results of the literatures [20,25]. However, the results differed from the reports of studies [26,27] employed different methods of GO synthesis and the examined different cell lines.

\section{Conclusion}

The present study investigated the cytotoxicity of GO and its possible mechanisms using human multiple myeloma RPMI 8226 cells as a cell model. CCK- 8 assay data elucidated that GO could cause cytotoxicity in multiple myeloma cells. Moreover, GO induces cytotoxicity in a dose-dependent manner, but displays low cytotoxicity at concentrations below $100 \mathrm{mg} / \mathrm{L}$. GO does not induce apoptosis. The generation of oxidative stress is the primary cytotoxic mechanism of GO. Although the clinical significance of our results remains to be elucidated, our study offers a sound foundation for the use of GO-based nano-drugs in the treatment of hematological malignancies.

\section{Acknowledgement}

This work was supported by Science \& Technology Development Program of Shandong Province, China (2012GSF111819).

\section{References}

[1] J. Kim, L.J. Cote and J. Huang, Two dimensional soft material: new faces of graphene oxide, Acc. Chem. Res. 45 (2012), 1356-1364. 
[2] O. Akhavan, E. Ghaderi and H. Emamy, Nontoxic concentrations of PEGylated graphene nanoribbons for selective cancer cell imaging and photothermal therapy, J. Mater. Chem. 22 (2012), 20626-20633.

[3] Y. Wang, Z. Li, D. Hu, C.T. Lin, J. Li and Y. Lin, Aptamer/Graphene oxide nanocomplex forin situ molecular probing in living cells, J. Am. Chem. Soc. 132 (2010), 9274-9276.

[4] N. Mohanty and V. Berry, Graphene-based single-bacterium resolution biodevice and DNA transistor: Interfacing graphene derivatives with nanoscale and microscale biocomponents, Nano. Lett. 8 (2008), 4469-4476.

[5] K.P. Liu, J.J. Zhang, F.F. Cheng, T.T Zheng, C.M. Wang and J.J. Zhu, Green and facile synthesis of highly biocompatible graphene nanosheets and its application for cellular imaging and drug delivery, J. Mater. Chem. 21 (2011), 12034-12040.

[6] Y.N. Ni, F.Y Zhang and S. Kokot, Graphene oxide as a nanocarrier for loading and delivery of medicinal drugs and as a biosensor for detection of serum albumin, Anal. Chim. Acta. 769 (2013), 40-48.

[7] N. Saikia and R.C. Deka, Ab initio study on the noncovalent adsorption of camptothecin anticancer drug onto graphene, defect modified graphene and graphene oxide, J. Comput. Aided Mol. Des. 27 (2013), 807-821.

[8] S.L. Wu, X.D. Zhao, Y.H. Li, Q.J. Du, J.K. Sun, Y.H. Wang, X. Wang, Y.Z. Xia, Z.H. Wang and L.H. Xia, Adsorption properties of doxorubicin hydrochloride onto graphene oxide: equilibrium, kinetic and thermodynamic studies, Materials 6 (2013), 2026-2042.

[9] S. Marchesan and M. Prato, Nanomaterials for (Nano) medicine, ACS Med. Chem. Lett. 4 (2013), 147-149.

[10] S. Marchesan, M. Melchionna and M. Prato, Carbon nanostructures for nanomedicine: opportunities and challenges, Fullerenes Nanotubes and Carbon Nanostructures 22 (2014), 190-195.

[11] X.Q. Zhang, X. Xu, N. Bertrand, E. Pridgen, A.Swami and O.C. Farokhzad, Interactions of nanomaterials and biological systems: Implications to personalized nanomedicine, Adv. Drug Deliv. Rev. 64 (2012), 1363-1384.

[12] O. Akhavan, E. Ghaderi, H. Emamy and F. Akhavan, Genotoxicity of graphene nanoribbons inhuman mesenchymal stem cells, Carbon 54 (2013), 419-431.

[13] K. Wang, J. Ruan, H, Song, J.L. Zhang, Y. Wo, S.W. Guo and D.X. Cui, Biocompatibility of graphene oxide, Nanoscale Res. Lett. 6 (2011), 1-8.

[14] A. Wang, K. Pu, B. Dong, Y. Liu, L. Zhang, Z. Zhang, W. Duan and Y. Zhu, Role of surface charge and oxidative stress in cytotoxicity and genotoxicity of graphene oxide towards human lung fibroblast cells, J. Appl. Toxicol. 33 (2013), 1156-1164.

[15] K.H. Liao, Y.S. Lin, C.W. Macosko and C. Haynes, Cytotoxicity of graphene oxide and graphene in human erythrocytes and skin fibroblasts, ACS Appl. Mater. Interfaces 3 (2011), 2607-2615.

[16] A. Sasidharan, L.S. Panchakarla, P. Chandran, D.Menon, S. Nair, C.N. Rao and M. Koyakutty, Differential nano-bio interactions and toxicity effects of pristine versus functionalized graphene, Nanoscale 3 (2011), 2461-2464.

[17] O. Akhavan, E. Ghaderi and A. Akhavan, Size-dependent genotoxicity of graphene nanoplatelets in human stem cells, Biomaterials 33 (2012), 8017-8025.

[18] H. Yue, W. Wei, Z.G. Yue, B. Wang, N.N. Luo, Y.J. Gao, D. Ma, G. Ma and Z.G. Su, The role of the lateral dimension of graphene oxide in the regulation of cellular responses, Biomaterials 33 (2012), 4013-4021.

[19] S. Mullick Chowdhury, G. Lalwani, K. Zhang, J.Y. Yang, K. Neville and B. Sitharaman, Cell specificcytotoxicity and uptake of graphene nanoribbons, Biomaterials 34 (2013), 283-293.

[20] Y. Chang, S.T. Yang, J.H. Liu, E. Dong, Y. Wang, A. Cao, Y. Liu and H. Wang, In vitro toxicity evaluation of graphene oxide on A549 cells, Toxicol. Lett. 200 (2011), 201-210.

[21] T. Lammel, P. Boisseaux, M.L. Fernández-Cruz and J.M. Navas, Internalization and cytotoxicity of graphene oxide and carboxyl graphene nanoplatelets in the human hepatocellular carcinoma cell line Hep G2, Part Fibre Toxicol. 10 (2013), 27.

[22] Y. Zhang, S.F. Ali, E. Dervishi, Y. Xu, Z. Li, D. Casciano and A.S. Biris, Cytotoxicity effects of graphene and single-wall carbon nanotubes in neural phaeochromocytoma-derived PC12 cells, ACS Nano. 4 (2010), 3181-3186.

[23] J. Ahmad, M. Ahamed, M.J. Akhtar, S.A. Alrokayan, M.A. Siddiqui, J. Musarrat and A.A. Al-Khedhairy, Apoptosis induction by silica nanoparticles mediated through reactive oxygen species in human liver cell line HepG2, Toxicol. Appl. Pharmacol. 259 (2012), 160-168.

[24] S. Gurunathan, J.W. Han, V. Eppakayala and J.H. Kim, Green synthesis of graphene and its cytotoxic effects in human breast cancer cells, Int. J. Nanomedicine 8 (2013), 1015-1027.

[25] M. Lv, Y. Zhang, L. Liang, M. Wei, W. Hu and X. Li, Effect of graphene oxide on undifferentiated and retinoic acid-differentiated SH-SY5Y cells line, Nanoscale 4 (2012), 3861-3866.

[26] G. Qu, S. Liu, S. Zhang, L. Wang, X. Wang, B. Sun, N. Yin and X. Gao, Graphene oxide induces toll-like receptor 4 (TLR4)-dependent necrosis in macrophages, ACS Nano. 7 (2013), 5732-5745.

[27] N.V. Vallabani, S. Mittal, R.K. Shukla, A.K. Pandey, S.R. Dhakate and R. Pasricha, Toxicity of graphene in normal human lung cells (BEAS-2B), J. Biomed. Nanotechno. 7 (2011), 106-107. 